\title{
CORRECTIONS
}

\section{A student with macrocytic anaemia}

Owing to an editing error, the short answer to question " 2 " in this Endgames quiz (BMJ 2014;348:g3099, doi:10.1136/bmj. g3099) contains an inaccuracy. This answer should have read: "In view of her diet and short history, folate or vitamin B12 deficiency, with folate deficiency [not "vitamin B12 deficiency"] being the most likely." This error was corrected for the print version of the article.

Cite this as: BMJ 2014;348:93399

๑ BMJ Publishing Group Ltd 2014 